\title{
Dynamical Behavior and the Classification of Single Traveling Wave Solutions for the Coupled Nonlinear Schrödinger Equations with Variable Coefficients
}

\author{
Zhao Li $\mathbb{D}^{1,2}$ Peng Li, ${ }^{3}$ and Tianyong Han ${ }^{2}$ \\ ${ }^{1}$ College of Computer Science, Chengdu University, Chengdu 610106, China \\ ${ }^{2}$ Key Laboratory of Pattern Recognition and Intelligent Information Processing, Institutions of Higher Education of \\ Sichuan Province, Chengdu University, Chengdu 610106, China \\ ${ }^{3}$ North China Electric Power Test and Research Institute, China Datang Corporation Science and Technology Research Institute Co., \\ Beijing 100040, China
}

Correspondence should be addressed to Zhao Li; lizhao10.26@163.com

Received 21 June 2021; Revised 3 August 2021; Accepted 11 August 2021; Published 27 August 2021

Academic Editor: Ming Mei

Copyright (@) 2021 Zhao Li et al. This is an open access article distributed under the Creative Commons Attribution License, which permits unrestricted use, distribution, and reproduction in any medium, provided the original work is properly cited.

In this paper, the dynamical properties and the classification of single traveling wave solutions of the coupled nonlinear Schrödinger equations with variable coefficients are investigated by utilizing the bifurcation theory and the complete discrimination system method. Firstly, coupled nonlinear Schrödinger equations with variable coefficients are transformed into coupled nonlinear ordinary differential equations by the traveling wave transformations. Then, phase portraits of coupled nonlinear Schrödinger equations with variable coefficients are plotted by selecting the suitable parameters. Furthermore, the traveling wave solutions of coupled nonlinear Schrödinger equations with variable coefficients which correspond to phase orbits are easily obtained by applying the method of planar dynamical systems, which can help us to further understand the propagation of the coupled nonlinear Schrödinger equations with variable coefficients in nonlinear optics. Finally, the periodic wave solutions, implicit analytical solutions, hyperbolic function solutions, and Jacobian elliptic function solutions of the coupled nonlinear Schrödinger equations with variable coefficients are constructed.

\section{Introduction}

The coupled nonlinear Schrödinger (CNLS) equations [1-3] are a very important mathematical physical model in the fields of quantum mechanics, nonlinear optics, optical fiber communication, Bose-Einstein condensate, fluid mechanics, and so on. Because of its importance, many new methods and techniques have been proposed to study the CNLS equation, which include the Bäcklund transformation, the variational iteration method, the extended unified method, and the Hirota method [4-9]. However, the study of CNLS equations with variable coefficients [10-14] has more important theoretical significance and practical value than CNLS equations with constant coefficients because it usually describes the inhomogeneous effects of nonlinear optical pulse propa- gations in the real optical fiber communication system. The CNLS equations with variable coefficients are expressed as follows:

$$
\left\{\begin{array}{l}
i \frac{\partial \psi}{\partial x}+\beta(x) \frac{\partial^{2} \psi}{\partial t^{2}}+2 g(x)\left(|\psi|^{2}+|\phi|^{2}\right) \psi=0 \\
i \frac{\partial \phi}{\partial x}+\beta(x) \frac{\partial^{2} \phi}{\partial t^{2}}+2 g(x)\left(|\psi|^{2}+|\phi|^{2}\right) \phi=0
\end{array}\right.
$$

where $\psi=\psi(t, x)$ and $\phi=\phi(t, x)$ denote the slowly varying amplitudes of two orthogonally polarized optical pluses, which are complex-valued functions with the retarded time $t$ and the normalized propagation distance $x .(t, x) \in[0, T]$ $\times[a, b], T \geq 0,-\infty \leq a \leq b \leq+\infty$. The coefficients $\beta(x)$ and $g$ 
$(x)$ are integrable real functions of $x$, which represent the group velocity dispersion and the Kerr nonlinearity, respectively. Obviously, the integrability method, the modified sine-Gordon equation method, the unified method, the improved F-expansion method, and the Hirota bilinear method have used to establish the traveling wave solutions of the CNLS equation with variable coefficients in Ref. [1014], respectively. In this paper, we investigate the dynamical properties and the classification of single traveling wave solutions of the CNLS equation with variable coefficients based on the bifurcation theory and the complete discrimination system method.

The synopsis of the article is organized as follows. In Section 2, the bifurcation theory is employed to investigate the CNLS equations with variable coefficients; phase portraits and some Jacobian elliptic function solutions are obtained. In Section 3, some single traveling wave solutions of the CNLS equations with variable coefficients are obtained by using the complete discrimination system method. In Section 4 , we present some research results.

\section{Bifurcations of Phase Portraits of System (9)}

In order to analyze the dynamic behavior of Equation (1), we first introduce the following traveling wave transformation:

$\psi(t, x)=\Psi(\xi) e^{i \eta}, \phi(t, x)=\Phi(\xi) e^{i \eta}, \xi=\mu(t-\lambda(x)), \eta=\alpha t-\theta(x)$

where $\Psi$ and $\Phi$ are a real valued function, $\mu$ and $\alpha$ are arbitrary constants, and $\lambda(x)$ and $\theta(x)$ are arbitrary function of $x$

Substituting (2) into (1), we get the coupled nonlinear differential equations

$$
\begin{aligned}
\beta(x) \mu^{2} \Psi^{\prime \prime}(\xi) & +i \mu\left(2 \alpha \beta(x)-\lambda^{\prime}(x)\right) \Psi^{\prime}(\xi) \\
& +\left(\theta^{\prime}(x)-\alpha^{2} \beta(x)\right) \Psi(\xi) \\
& +2 g(x)\left(\Psi^{2}(\xi)+\Phi^{2}(\xi)\right) \Psi(\xi)=0 \\
\beta(x) \mu^{2} \Phi^{\prime \prime}(\xi)+ & i \mu\left(2 \alpha \beta(x)-\lambda^{\prime}(x)\right) \Phi^{\prime}(\xi) \\
+ & \left(\theta^{\prime}(x)-\alpha^{2} \beta(x)\right) \Phi(\xi) \\
+ & 2 g(x)\left(\Psi^{2}(\xi)+\Phi^{2}(\xi)\right) \Phi(\xi)=0 .
\end{aligned}
$$

Next, we decompose real parts and imaginary parts of (3) and put zero for imaginary parts of Equations (3), where the imaginary part of Equations (3) is $\lambda^{\prime}(x)=2 \alpha \beta(x)$. Then, we set $\lambda(x)=2 \alpha \int_{a}^{x} \beta(\varsigma) d \varsigma, a \leq x \leq b$. Therefore, $\xi=\mu(t-2 \alpha$ $\int_{a}^{x} \beta(\varsigma) d \varsigma$ ). Using the coefficients of $\Psi^{\prime \prime}$ (or $\Phi^{\prime \prime}$ ) as the normalization coefficient, we have

$$
\theta(x)=\int_{a}^{x}\left(\mu^{2} c_{1}+\alpha^{2}\right) \beta(\varsigma) d \varsigma+\theta_{0}, \quad g(x)=\frac{c_{2}}{2} \mu^{2} \beta(x),
$$

$$
\lambda(x)=2 \alpha \int_{a}^{x} \beta(\varsigma) d \varsigma,
$$

where $c_{1}$ and $c_{2}$ are constants and $\theta_{0}$ is the integral constant. On the other hand, the real parts of Equations (3) are simplified as

$$
\begin{aligned}
& \Psi^{\prime \prime}(\xi)+c_{1} \Psi(\xi)+c_{2}\left(\Psi^{2}(\xi)+\Phi^{2}(\xi)\right) \Psi(\xi)=0 \\
& \Phi^{\prime \prime}(\xi)+c_{1} \Phi(\xi)+c_{2}\left(\Psi^{2}(\xi)+\Phi^{2}(\xi)\right) \Phi(\xi)=0
\end{aligned}
$$

Let $d \Psi / d \xi=p, d \Phi / d \xi=q$; system (5) is transformed into a four-dimensional dynamical system as follows:

$$
\left\{\begin{array}{l}
\frac{d \Psi}{d \xi}=p \\
\frac{d p}{d \xi}=-c_{2} \Psi^{3}(\xi)-c_{2} \Psi(\xi) \Phi^{2}(\xi)-c_{1} \Psi(\xi) \\
\frac{d \Phi}{d \xi}=q \\
\frac{d q}{d \xi}=-c_{2} \Phi^{3}(\xi)-c_{2} \Psi^{2}(\xi) \Phi(\xi)-c_{1} \Phi(\xi) .
\end{array}\right.
$$

In order to remove the coupled relationship of (5), we , consider the dynamical behavior of system (6) in the submanifold $\Psi-l \Phi=0(l \neq 0)$ of the 4-dimensional phase space ( $\Psi, p, \Phi, q)$. Substituting $\Psi=l \Phi$ (or $\Phi=\Psi / l$ ) into (6), we obtain two completely uncoupled planar dynamical systems:

$$
\begin{aligned}
& \left(\begin{array}{l}
\frac{d \Psi}{d \xi}=p, \\
\frac{d p}{d \xi}=-\frac{c_{2}\left(1+l^{2}\right)}{l^{2}} \Psi^{3}(\xi)-c_{1} \Psi(\xi),
\end{array}\right. \\
& \left(\begin{array}{l}
\frac{d \Phi}{d \xi}=q, \\
\frac{d q}{d \xi}=-c_{2}\left(1+l^{2}\right) \Phi^{3}(\xi)-c_{1} \Phi(\xi) .
\end{array}\right.
\end{aligned}
$$

Systems (7) and (8) are the same if and only if they satisfy $l= \pm 1$. Then, system (7) can be rewritten as the following planar dynamical system

$$
\left(\begin{array}{l}
\frac{d \Psi}{d \xi}=p \\
\frac{d p}{d \xi}=-2 c_{2} \Psi^{3}-c_{1} \Psi
\end{array}\right.
$$

which is equivalent to the following Hamiltonian system:

$$
H(p, \Psi)=\frac{1}{2} p^{2}+\frac{c_{2}}{2} \Psi^{4}+\frac{c_{1}}{2} \Psi^{2}=h, \quad h \in \mathbb{R} .
$$

Suppose $f(\Psi)=-2 c_{2} \Psi^{3}-c_{1} \Psi$, and further assume that $M_{i}\left(\Psi_{i}, 0\right)(i=0,1,2)$ is an equilibrium points of system (9), 
then the eigenvalues of system (9) at the equilibrium points are expressed as $\lambda_{1,2}= \pm \sqrt{f^{\prime}(\Psi)}$. Thus, we can easily get three zeros of $f(\Psi)$ when $c_{1} c_{2}<0$, which are $\Psi_{0}=0, \Psi_{1}=$ $-\sqrt{-c_{1} / 2 c_{2}}$ and $\Psi_{2}=\sqrt{-c_{1} / 2 c_{2}}$. We can easily obtain one zero of $f(\Psi)$ when $c_{1} c_{2}>0$, which is $\Psi_{3}=0$. By the bifurcation theory of planar dynamical systems, the equilibrium point $M_{i}\left(\Psi_{i}, 0\right)$ is a saddle point when $f^{\prime}\left(\Psi_{i}\right)>0$, the equilibrium point $M_{i}\left(\Psi_{i}, 0\right)$ is a degraded saddle point when $f^{\prime}$ $\left(\Psi_{i}\right)=0$, and the equilibrium point $M_{i}\left(\Psi_{i}, 0\right)$ is center point when $f^{\prime}\left(\Psi_{i}\right)<0$. The phase portraits of (9) depending on different parameters $c_{1}$ and $c_{2}$ are shown in Figure 1.

Lemma 1 (see [15]). Let $\Psi(\xi)$ be a continuous solution of system (9) for $\xi \in(-\infty,+\infty)$ and $\lim _{\xi \rightarrow \infty} \Psi(\xi)=m$, $\lim _{\xi \longrightarrow-\infty} \Psi(\xi)=n$ :

(1) $\Psi(\xi)$ is called a smooth solitary wave solution if $m=n$ . Usually, a solitary wave solution of Equation (1) corresponds to a smooth homoclinic orbit of system (7)

(2) $\Psi(\xi)$ is called a smooth kink or antikink solution if $m \neq n$. A smooth kink (or antikink) wave solution of Equation (1) corresponds to a smooth heteroclinic orbit (or a so-called connecting orbit) of system (7)

Remark 2. Obviously (see [16-20]), all traveling wave solutions of (1) can be obtained from the phase orbits of the Hamiltonian system (9) according to Lemma 1.

\subsection{The Case $c_{1}>0$ and $c_{2}<0$}

(1) For $h \in\left(0,-c_{1}^{2} / 8 c_{2}\right)$, the system (10) can be written as

$$
p^{2}=-c_{2}\left(\Psi^{4}+\frac{c_{1}}{c_{2}} \Psi^{2}-\frac{2 h}{c_{2}}\right)=-c_{2}\left(\Psi_{1 h}^{2}-\Psi^{2}\right)\left(\Psi_{2 h}^{2}-\Psi^{2}\right),
$$

where $\Psi_{1 h}^{2}=-c_{1} / 2 c_{2}-1 / 2 c_{2} \sqrt{c_{1}^{2}+8 c_{2} h}$ and $\Psi_{2 h}^{2}=-c_{1} / 2 c_{2}$ $+1 / 2 c_{2} \sqrt{c_{1}^{2}+8 c_{2} h}$.

Substituting (11) into the first equation of system (9), integrating them along the periodic orbits, then we obtain

$$
\Psi_{1}(\xi)= \pm \Psi_{1 h} \operatorname{sn}\left(\Psi_{2 h} \sqrt{-c_{2}}\left(\xi-\xi_{0}\right), \frac{\Psi_{1 h}}{\Psi_{2 h}}\right)
$$

Namely,

$$
\begin{aligned}
\psi_{1}(t, x)= & \pm \Psi_{1 h} \operatorname{sn}\left(\Psi _ { 2 h } \sqrt { - c _ { 2 } } \left(\mu\left(t-2 \alpha \int_{a}^{x} \beta(\varsigma) d \varsigma\right)\right.\right. \\
& \left.\left.-\xi_{0}\right), \frac{\Psi_{1 h}}{\Psi_{2 h}}\right) e^{i\left(\alpha t-\int_{a}^{x}\left(\mu^{2} c_{1}+\alpha^{2}\right) \beta(\varsigma) d \varsigma+\theta_{0}\right)} .
\end{aligned}
$$

(2) For $h=-c_{1}^{2} / 8 c_{2}$, we easily obtain two kink solitary wave solutions

$$
\begin{gathered}
\Psi_{2}(\xi)= \pm \sqrt{-\frac{c_{1}}{2 c_{2}}} \tanh \left(\sqrt{-c_{2}}\left(\xi-\xi_{0}\right)\right) \\
\psi_{2}(t, x)= \pm \sqrt{-\frac{c_{1}}{2 c_{2}}} \tanh \left(\sqrt { - c _ { 2 } } \left(\mu\left(t-2 \alpha \int_{a}^{x} \beta(\varsigma) d \varsigma\right)\right.\right. \\
\left.\left.-\xi_{0}\right)\right) e^{i\left(\alpha t-\int_{a}^{x}\left(\mu^{2} c_{1}+\alpha^{2}\right) \beta(\varsigma) d \varsigma+\theta_{0}\right)} .
\end{gathered}
$$

When $\mu=2, c_{1}=2, c_{2}=-1, \alpha=1 / 2$, and $\xi_{0}=0$, the dark soliton solution $\left|\psi_{2}\right|$ of Equation (1) is drawn in Figure 2, where $\beta(x)=1$ and $\beta(x)=\sin x$, respectively.

\subsection{The Case $c_{1}<0$ and $c_{2}>0$}

(1) For $h \in\left(-c_{1}^{2} / 8 c_{2}, 0\right)$, the system (10) can be written as

$$
p^{2}=c_{2}\left(-\Psi^{4}-\frac{c_{1}}{c_{2}} \Psi^{2}+\frac{2 h}{c_{2}}\right)=c_{2}\left(\Psi^{2}-\Phi_{1 h}^{2}\right)\left(\Psi_{2 h}^{2}-\Psi^{2}\right),
$$

where $\Psi_{1 h}^{2}=-c_{1} / 2 c_{2}-\left(1 / 2 c_{2}\right) \sqrt{c_{1}^{2}+8 c_{2} h}, \Psi_{2 h}^{2}=-c_{1} / 2 c_{2}+1$ $/ 2 c_{2}\left(1 / 2 c_{2}\right) \sqrt{c_{1}^{2}+8 c_{2} h}$.

Substituting (16) into $d \Psi / d \xi=p$, we can obtain the smooth periodic wave solution

$$
\begin{aligned}
\Psi_{3}(\xi) & = \pm \Psi_{2 h} \operatorname{dn}\left(\Psi_{2 h} \sqrt{c_{2}}\left(\xi-\xi_{0}\right), \frac{\sqrt{\Psi_{2 h}^{2}-\Psi_{1 h}^{2}}}{\Psi_{2 h}}\right), \\
\psi_{3}(t, x)= & \pm \Psi_{2 h} \operatorname{dn}\left(\Psi _ { 2 h } \sqrt { c _ { 2 } } \left(\mu\left(t-2 \alpha \int_{a}^{x} \beta(\varsigma) d \varsigma\right)\right.\right. \\
& \left.\left.-\xi_{0}\right), \frac{\sqrt{\Psi_{2 h}^{2}-\Psi_{1 h}^{2}}}{\Psi_{2 h}}\right) e^{i\left(\alpha t-\int_{a}^{x}\left(\mu^{2} c_{1}+\alpha^{2}\right) \beta(\varsigma) d \varsigma+\theta_{0}\right)} .
\end{aligned}
$$

(2) For $h=0$, we obtain two bell solitary wave solutions:

$$
\begin{gathered}
\Psi_{4}(\xi)= \pm \sqrt{-\frac{c_{1}}{c_{2}}} \operatorname{sech}\left(\sqrt{-c_{1}}\left(\xi-\xi_{0}\right)\right), \\
\psi_{4}(t, x)= \pm \sqrt{-\frac{c_{1}}{c_{2}}} \operatorname{sech}\left(\sqrt { - c _ { 1 } } \left(\mu\left(t-2 \alpha \int_{a}^{x} \beta(\varsigma) d \varsigma\right)\right.\right. \\
\left.\left.-\xi_{0}\right)\right) e^{i\left(\alpha t-\int_{a}^{x}\left(\mu^{2} c_{1}+\alpha^{2}\right) \beta(\varsigma) d \varsigma+\theta_{0}\right)} .
\end{gathered}
$$




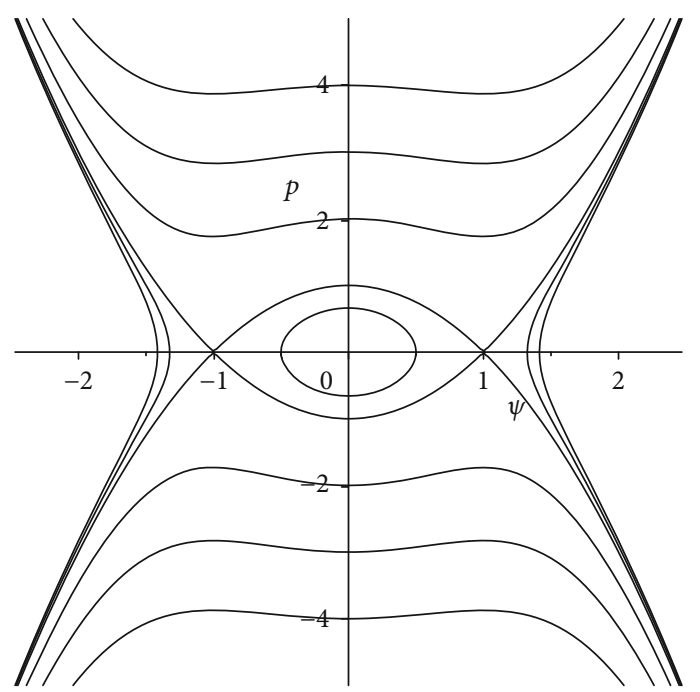

(a)

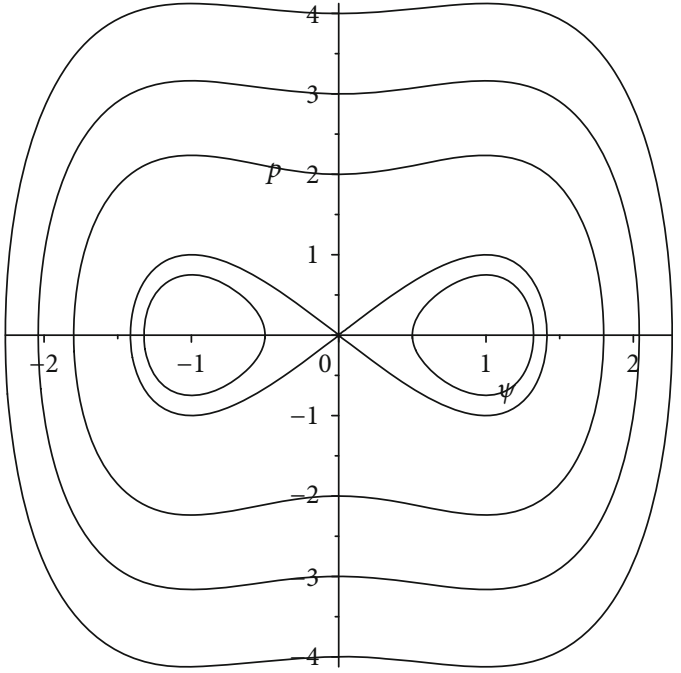

(b)

FIgURE 1: The phase portraits of system (9).

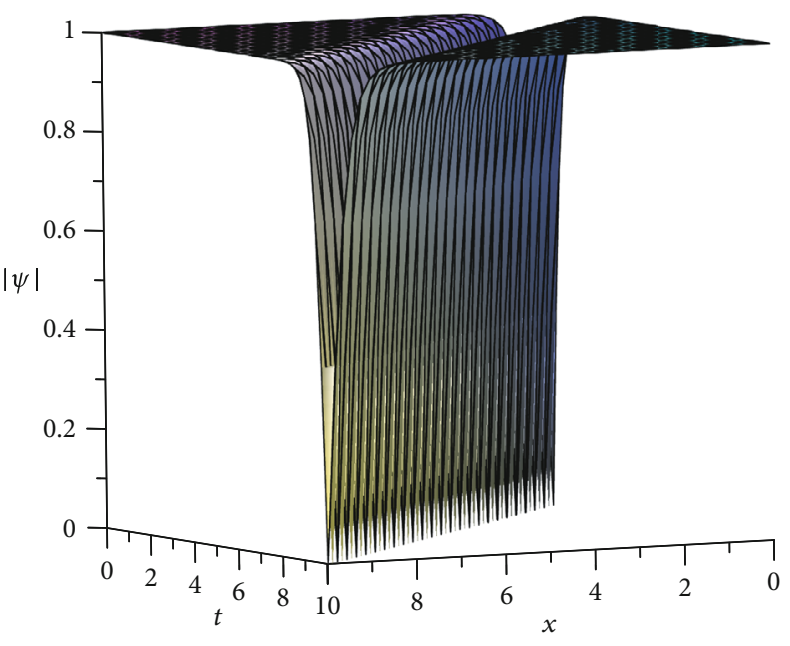

(a)

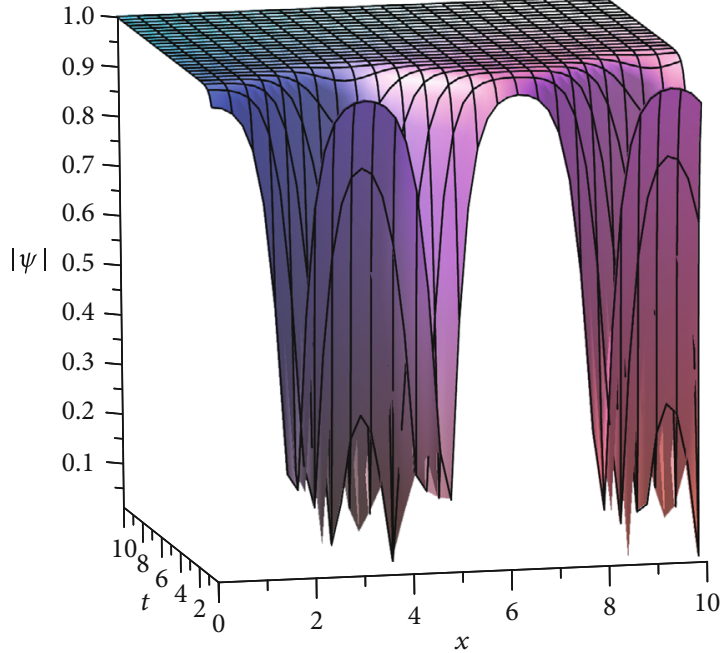

(b)

FIgURE 2: The dark solitary wave solution $\left|\psi_{2}(t, x)\right|$ of Equation (15) for differential parameter $\beta(x)$.

When $\mu=2, c_{1}=-2, c_{2}=1, \alpha=1 / 2, \xi_{0}=0$, the bright solitary wave solution $\left|\psi_{4}\right|$ of Equation (1) is drawn in Figure 3 , where $\beta(x)=e^{x}$ and $\beta(x)=e^{x}+2 x e^{x^{2}}$, respectively.

(3) For $h \in(0,+\infty)$, the system (10) can be written as

$$
p^{2}=c_{2}\left(-\Psi^{4}-\frac{c_{1}}{c_{2}} \Psi^{2}+\frac{2 h}{c_{2}}\right)=c_{2}\left(\Psi_{3 h}^{2}+\Psi^{2}\right)\left(\Psi_{4 h}^{2}-\Psi^{2}\right),
$$

where $\Psi_{3 h}^{2}=-c_{1} / 2 c_{2}+1 / 2 c_{2} \sqrt{c_{1}^{2}+8 c_{2} h}$ and $\Psi_{4 h}^{2}=c_{1} / 2 c_{2}+1$ $12 c_{2} \sqrt{c_{1}^{2}+8 c_{2} h}$.
Substituting (20) into $d \Psi / d \xi=p$ and integrating them, we obtain two families of periodic solutions

$$
\Psi_{5}(\xi)=\Psi_{4 h} \mathrm{cn}\left(\sqrt{c_{2}\left(\Psi_{3 h}^{2}+\Psi_{4 h}^{2}\right)}\left(\xi-\xi_{0}\right), \frac{\Psi_{4 h}}{\sqrt{\Psi_{3 h}^{2}+\Psi_{4 h}^{2}}}\right)
$$

Namely,

$$
\begin{aligned}
\psi_{5}(t, x)= & \Psi_{4 h} \operatorname{cn}\left(\sqrt { c _ { 2 } ( \Psi _ { 3 h } ^ { 2 } + \Psi _ { 4 h } ^ { 2 } ) } \left(\mu\left(t-2 \alpha \int_{a}^{x} \beta(\varsigma) d \varsigma\right)\right.\right. \\
& \left.\left.-\xi_{0}\right), \frac{\Psi_{4 h}}{\sqrt{\Psi_{3 h}^{2}+\Psi_{4 h}^{2}}}\right) e^{i\left(\alpha t-\int_{a}^{x}\left(\mu^{2} c_{1}+\alpha^{2}\right) \beta(\varsigma) d \varsigma+\theta_{0}\right)} .
\end{aligned}
$$




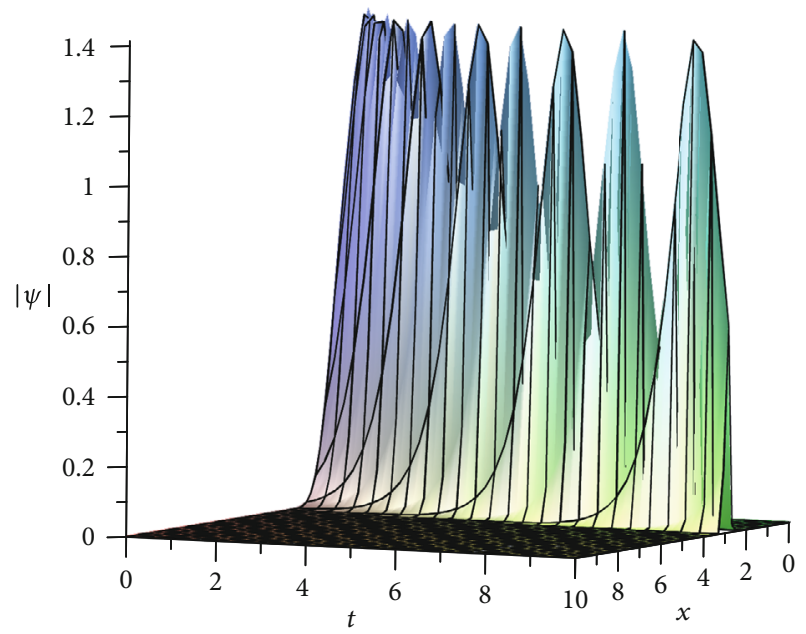

(a)

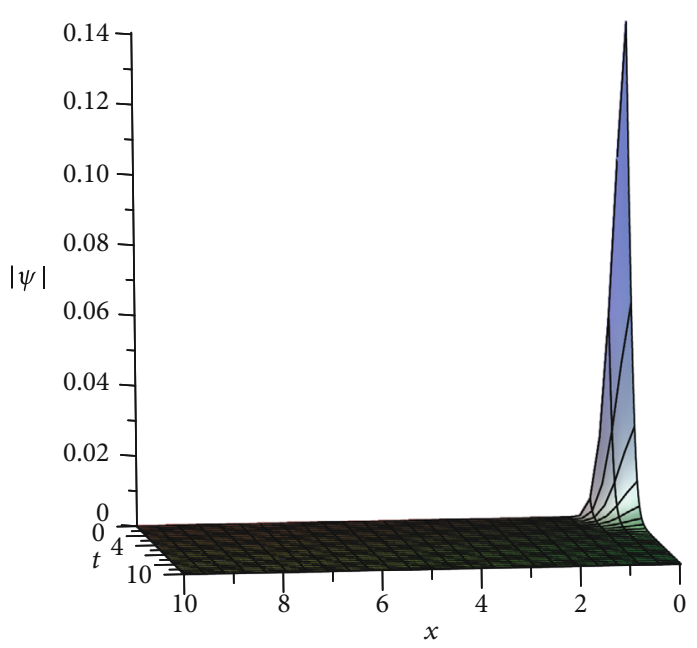

(b)

FIgURE 3: The dark solitary wave solution $\left|\psi_{4}(t, x)\right|$ of Equation (19) for differential parameter $\beta(x)$.

Remark 3. In this section, we mainly discuss the solution $\psi$ $(t, x)$ of Equation (1) when $c_{1} c_{2}<0$. Moreover, we can easily obtain the solution $\phi(t, x)$ of Equation (1) through their relationship $\Psi=l \Phi$.

\section{The Classification of Single Traveling Wave Solutions via Complete Discrimination Systems}

In order to remove the coupled relationship of (5), we assume $\Psi=l \Phi(l \neq 0)$. Substituting $\Psi=l \Phi$ into (5), we have

$$
\Psi^{\prime \prime}+c_{1} \Psi+c_{2} \Psi^{2}+\frac{c_{1}}{l^{2}} \Psi^{3}=0
$$

Multiplying both sides of (23) by $\Psi^{\prime}$ and integrating once with respect to $\xi$, we have

$$
\left(\Psi^{\prime}\right)^{2}=-\frac{c_{2}}{2 l^{2}} \Psi^{4}-\frac{2 c_{2}}{3} \Psi^{3}-c_{1} \Psi^{2}-2 c_{0}
$$

where $c_{0}$ is the integral constant.

Make the transformation

$$
\varphi=\left(-\frac{c_{2}}{2 l^{2}}\right)^{1 / 4}\left(\Psi+\frac{l^{2}}{3}\right), \xi_{1}=\left(-\frac{c_{2}}{2 l^{2}}\right)^{1 / 4} \xi .
$$

Next, substituting (25) into (24), it becomes

$$
\left(\varphi_{\xi_{1}}^{\prime}\right)^{2}=\varphi^{4}+a_{2} \varphi^{2}+a_{1} \varphi+a_{0}
$$

where $\quad a_{2}=-2 c_{1}|l| / \sqrt{-2 c_{2}}, \quad a_{1}=\left(-16 l^{4} / 27 c_{2}+c_{1} l^{2} / 3\right)$ $\left(-c_{2} / 2 l^{2}\right)^{-1 / 4}$, and $a_{0}=c_{2} l^{6} / 54-c_{1} l^{4} / 9-2 c_{0}$. The solution of (26) can be written as the integral form

$$
\int \frac{d \varphi}{\sqrt{\varphi^{4}+a_{2} \varphi^{2}+a_{1} \varphi+a_{0}}}= \pm\left(\xi_{1}-\xi_{0}\right)
$$

where $\xi_{0}$ is an arbitrary constant.

Let $G(\varphi)=\varphi^{4}+a_{2} \varphi^{2}+a_{1} \varphi+a_{0}$. Thus, we can obtain its complete discrimination system [21-23] as follows:

$$
\begin{gathered}
D_{1}=4, D_{2}=-\mathrm{b}_{1}, D_{3}=-2 b_{1}^{3}+8 b_{1} b_{3}-9 b_{2}^{2}, E_{2}=9 b_{1}^{2}-32 b_{1} b_{3}, \\
D_{4}=-b_{1}^{3} b_{2}^{2}+4 b_{1}^{4} b_{3}+36 b_{1} b_{2}^{2} b_{3}-32 b_{1}^{2} b_{3}^{2}-\frac{27}{4} b_{2}^{4}+64 b_{3}^{3} .
\end{gathered}
$$

Case 1. When $D_{2}<0, D_{3}=0$, and $D_{4}=0$. Then, $G(\varphi)$ admits a pair of conjugate complex roots of multiplicities two, namely,

$$
G(\varphi)=\left[(\varphi-u)^{2}+v^{2}\right]^{2},
$$

where $v>0$. Substituting (29) into (27), we attain

$$
\xi_{1}-\xi_{0}=\int \frac{d \varphi}{(\varphi-u)^{2}+v^{2}}=\frac{1}{v} \arctan \frac{\varphi-u}{v},
$$

then the solution of (23) is expressed as follows:

$$
\Psi_{1}(\xi)= \pm\left(-\frac{c_{2}}{2 l^{2}}\right)^{-\frac{1}{4}} v \tan \left(v\left(\left(-\frac{c_{2}}{2 l^{2}}\right)^{\frac{1}{4}} \xi-\xi_{0}\right)\right)-\frac{l^{2}}{3} .
$$


Namely,

$$
\begin{aligned}
\psi_{1}(t, x)= & {\left[ \pm\left(-\frac{c_{2}}{2 l^{2}}\right)^{-1 / 4} v \tan \left(v \left(\left(-\frac{c_{2}}{2 l^{2}}\right)^{1 / 4} \mu(t\right.\right.\right.} \\
& \left.\left.-2 \alpha \int_{a}^{x} \beta(\varsigma) d \varsigma\right)-\xi_{0}\right) \\
& \left.-\frac{l^{2}}{3}\right] e^{i\left(\alpha t-\int_{a}^{x}\left(\mu^{2} c_{1}+\alpha^{2}\right) \beta(\varsigma) d \varsigma+\theta_{0}\right)} .
\end{aligned}
$$

Case 2. $D_{2}=0, D_{3}=0$, and $D_{4}=0 . G(\varphi)$ has real roots of multiplicities of four, namely, $G(\varphi)=\varphi^{4}$. Substituting $G(\varphi)$ $=\varphi^{4}$ into (27), we can get

$$
\xi_{1}-\xi_{0}=\int \frac{d \varphi}{\varphi^{2}}=-\frac{1}{\varphi},
$$

then the solutions of Equation (1) can be shown as

$$
\begin{aligned}
\psi_{2}(t, x)= & {\left[\mp\left(-\frac{c_{2}}{2 l^{2}}\right)^{-1 / 4} \frac{1}{\left(-c_{2} / 2 l^{2}\right)^{-1 / 4} \xi-\xi_{0}}\right.} \\
& \left.-\frac{l^{3}}{3}\right] e^{i\left(\alpha t-\int_{a}^{x}\left(\mu^{2} c_{1}+\alpha^{2}\right) \beta(s) d c+\theta_{0}\right)} .
\end{aligned}
$$

Case 3. $D_{2}>0, D_{3}=0, D_{4}=0$, and $E_{2}>0 . G(\varphi)$ has two real roots of multiplicities of two, namely,

$$
G(\varphi)=(\varphi-u)^{2}(\varphi-v)^{2},
$$

where $u>v$. Substituting (35) into (27), we can obtain

$$
\pm\left(\xi_{1}-\xi_{0}\right)=\int \frac{d \varphi}{(\varphi-u)(\varphi-v)}=\frac{1}{u-v} \ln \left|\frac{\varphi-u}{\varphi-v}\right| .
$$

When $\varphi>u$ or $\varphi<v$, we can obtain the solution of Equation (23) as follows:

$$
\begin{aligned}
\Psi_{3}\left(\xi_{1}\right) & =\frac{v-u}{e^{(u-v)\left(\xi_{1}-\xi_{0}\right)}-1}+v \\
& =\frac{v-u}{2}\left[\operatorname{coth} \frac{(u-v)\left(\xi_{1}-\xi_{0}\right)}{2}-1\right]+v,
\end{aligned}
$$

then we have

$$
\begin{aligned}
\psi_{3}(t, x)= & {\left[\frac{(v-u)\left(-c_{2} / 2 l^{2}\right)^{-1 / 4}}{2}\right.} \\
& \cdot\left(\operatorname{coth} \frac{(u-v)\left(\left(-c_{2} / 2 l^{2}\right)^{1 / 4} \mu\left(t-2 \alpha \int_{a}^{x} \beta(\varsigma) d \varsigma\right)-\xi_{0}\right)}{2}-1\right) \\
& \left.+v-\frac{l^{2}}{3}\right] e^{i\left(\alpha t-\int_{a}^{x}\left(\mu^{2} c_{1}+\alpha^{2}\right) \beta(\varsigma) d \varsigma+\theta_{0}\right)} .
\end{aligned}
$$

When $v<\Phi<u$, we can gain the solution of Equation (23) as follows:

$$
\begin{aligned}
\Psi_{4}\left(\xi_{1}\right) & =\frac{v-u}{-e^{(u-v)\left(\xi_{1}-\xi_{0}\right)}-1}+v \\
& =\frac{v-u}{2}\left[\tanh \frac{(u-v)\left(\xi_{1}-\xi_{0}\right)}{2}-1\right]+v .
\end{aligned}
$$

Similarly, we can have

$$
\begin{aligned}
\psi_{4}(t, x)= & {\left[\frac{(v-u)\left(-c_{2} / 2 l^{2}\right)^{-1 / 4}}{2}\right.} \\
& \cdot\left(\tanh \frac{(u-v)\left(\left(-c_{2} / 2 l^{2}\right)^{1 / 4} \mu\left(t-2 \alpha \int_{a}^{x} \beta(\varsigma) d \varsigma\right)-\xi_{0}\right)}{2}-1\right) \\
& \left.+v-\frac{l_{2}}{3}\right] e^{i\left(\alpha t-\int_{a}^{x}\left(\mu^{2} c_{1}+\alpha^{2}\right) \beta(\zeta) d c+\theta_{0}\right)} .
\end{aligned}
$$

Case 4. $D_{2}>0, D_{3}>0$, and $D_{4}=0 . G(\varphi)$ has two real roots and real roots with multiplicities of two, namely,

$$
G(\varphi)=\left(\varphi-\rho_{1}\right)^{2}\left(\varphi-\rho_{2}\right)\left(\varphi-\rho_{3}\right),
$$

where $\rho_{i}(i=1,2,3)$ are real numbers and $\rho_{2}>\rho_{3}$.

When $\rho_{1}>\rho_{2}$ and $\varphi<\rho_{2}$, or when $\rho_{1}<\rho_{3}$ and $\varphi<\rho_{3}$, we can have the implicit analytical solution of Equation (26):

$$
\begin{aligned}
\pm\left(\xi_{1}-\xi_{0}\right)= & \int \frac{d \varphi}{\sqrt{\left(\varphi-\rho_{1}\right)^{2}\left(\varphi-\rho_{2}\right)\left(\varphi-\rho_{3}\right)}} \\
= & \frac{1}{\left(\rho_{1}-\rho_{2}\right)\left(\rho_{1}-\rho_{3}\right)} \ln \\
& \cdot \frac{\left[\sqrt{\left(\varphi-\rho_{2}\right)\left(\rho_{1}-\rho_{3}\right)}-\sqrt{\left(\rho_{1}-\rho_{2}\right)\left(\varphi-\rho_{3}\right)}\right]^{2}}{\left|\varphi-\rho_{1}\right|} .
\end{aligned}
$$

When $\rho_{1}>\rho_{2}$ and $\varphi<\rho_{3}$, or when $\rho_{1}<\rho_{3}$ and $\varphi<\rho_{2}$, we can obtain the implicit analytical solution of Equation (26):

$$
\begin{aligned}
\pm\left(\xi_{1}-\xi_{0}\right)= & \int \frac{d \varphi}{\sqrt{\left(\varphi-\rho_{1}\right)^{2}\left(\varphi-\rho_{2}\right)\left(\varphi-\rho_{3}\right)}} \\
= & \frac{1}{\left(\rho_{1}-\rho_{2}\right)\left(\rho_{1}-\rho_{3}\right)} \ln \\
& \cdot \frac{\left[\sqrt{\left(\varphi-\rho_{2}\right)\left(\rho_{3}-\rho_{1}\right)}-\sqrt{\left(\rho_{2}-\rho_{1}\right)\left(\varphi-\rho_{3}\right)}\right]^{2}}{\left|\varphi-\rho_{1}\right|} .
\end{aligned}
$$


When $\rho_{2}>\rho_{1}>\rho_{3}$, we can have the implicit analytical solution of Equation (26):

$$
\begin{aligned}
\pm\left(\xi_{1}-\xi_{0}\right)= & \int \frac{d \varphi}{\sqrt{\left(\varphi-\rho_{1}\right)^{2}\left(\varphi-\rho_{2}\right)\left(\varphi-\rho_{3}\right)}} \\
= & \frac{1}{\left(\rho_{2}-\rho_{1}\right)\left(\rho_{1}-\rho_{3}\right)} \arcsin \\
& \cdot \frac{\left(\varphi-\rho_{2}\right)\left(\rho_{1}-\rho_{3}\right)+\left(\rho_{1}-\rho_{2}\right)\left(\varphi-\rho_{3}\right)}{\left|\left(\varphi-\rho_{1}\right)\left(\rho_{2}-\rho_{3}\right)\right|} .
\end{aligned}
$$

Case 5. $D_{2}>0, D_{3}=0, D_{4}=0$, and $E_{2}=0 . G(\varphi)$ has real roots of multiplicities of three and real roots with multiplicities of one, namely,

$$
G(\varphi)=(\varphi-u)^{3}(\varphi-\gamma)
$$

where $u$ and $v$ are real numbers. Substituting (45) into (27), we can yield

$$
\pm\left(\xi_{1}-\xi_{0}\right)=\int \frac{d \varphi}{\sqrt{(\varphi-u)^{3}(\varphi-v)}}=\frac{2}{v-u} \sqrt{\frac{\varphi-v}{\varphi-u}} .
$$

When $\varphi>u$ and $\varphi>v$, or when $\varphi<u$ and $\varphi<v$, the solution of Equation (26) is given by

$$
\varphi\left(\xi_{1}\right)=\frac{4(u-v)}{(v-u)^{2}\left(\xi_{1}-\xi_{0}\right)^{2}-4}+u
$$

then we can obtain a rational solution of Equation (1) as

$$
\begin{aligned}
\psi_{6}(t, x)= & {\left[\frac{4\left(-c_{2} / 2 l^{2}\right)^{-1 / 4}(u-v)}{\left(\left(-c_{2} / 2 l^{2}\right)^{1 / 4} \mu\left(t-2 \alpha \int_{a}^{x} \beta(\varsigma) d \varsigma\right)-\xi_{0}\right)^{2}(v-u)^{2}-4}\right.} \\
& \left.+u-\frac{l^{2}}{3}\right] e^{i\left(\alpha t-\int_{a}^{x}\left(\mu^{2} c_{1}+\alpha^{2}\right) \beta(\varsigma) d \varsigma+\theta_{0}\right)} .
\end{aligned}
$$

Case 6. $D_{2} D_{3}<0$ and $D_{4}=0 . G(\varphi)$ has real roots of multiplicities of two and a pair of conjugate complex roots, namely,

$$
G(\varphi)=\left(\varphi-\rho_{1}\right)^{2}\left[\left(\varphi-\rho_{2}\right)^{2}+\rho_{3}^{2}\right]
$$

where $\rho_{i}(i=1,2,3)$ are real numbers. Substituting (49) into (27), we can obtain

$$
\begin{aligned}
\pm\left(\xi_{1}-\xi_{0}\right) & =\int \frac{d \varphi}{\left(\varphi-\rho_{1}\right) \sqrt{\left(\varphi-\rho_{2}\right)^{2}+\rho_{3}^{2}}} \\
& =\frac{1}{\sqrt{\left(\rho_{1}-\rho_{2}\right)^{2}+\rho_{3}^{2}}} \ln \left|\frac{c_{1} \varphi+c_{2}-\sqrt{\left(\varphi-\rho_{2}\right)^{2}+\rho_{3}^{2}}}{\varphi-\rho_{1}}\right|
\end{aligned}
$$

where $\quad \varepsilon_{1}=\left(\rho_{1}-2 \rho_{2}\right) / \sqrt{\left(\rho_{1}-\rho_{2}\right)^{2}+\rho_{3}^{2}}, \quad \varepsilon_{2}=$ $\sqrt{\left(\rho_{1}-\rho_{2}\right)^{2}+\rho_{3}^{2}}-\rho_{1}\left(\rho_{1}-2 \rho_{2}\right) / \sqrt{\left(\rho_{1}-\rho_{2}\right)^{2}+\rho_{3}^{2}}$. Thus, we obtain the solution of Equation (1):

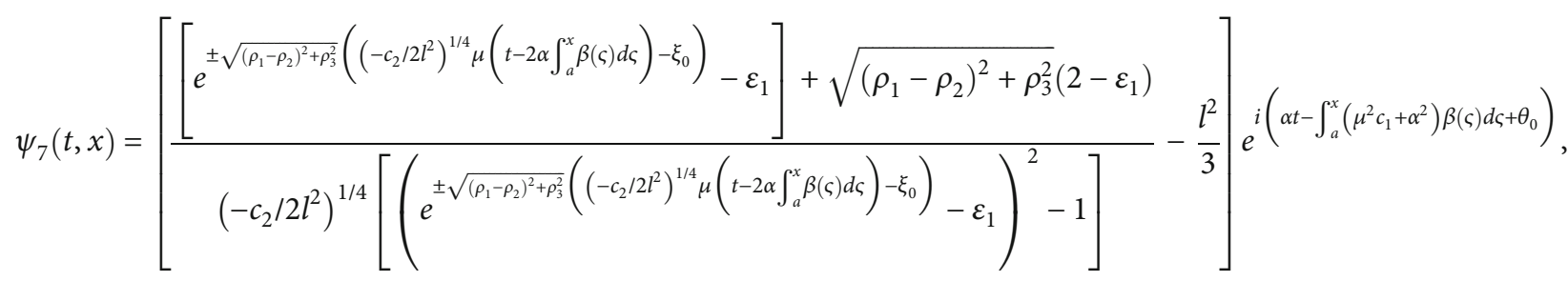

which is a solitary wave solution.

Case 7. $D_{2}>0, D_{3}>0$, and $D_{4}>0 . G(\varphi)$ has four distinct real roots, namely,

$$
G(\varphi)=\left(\varphi-\rho_{1}\right)\left(\varphi-\rho_{2}\right)\left(\varphi-\rho_{3}\right)\left(\varphi-\rho_{4}\right),
$$

where $\rho_{1}, \rho_{2}, \rho_{3}$, and $\rho_{4}$ are real numbers, and $\rho_{1}>\rho_{2}>\rho_{3}$ $>\rho_{4}$.

When $\varphi>\rho_{1}$ or $\varphi<\rho_{4}$, then we make the following transformation:

$$
\varphi=\frac{\rho_{2}\left(\rho_{1}-\rho_{4}\right) \sin ^{2} \theta-\rho_{1}\left(\rho_{2}-\rho_{4}\right)}{\left(\rho_{1}-\rho_{4}\right) \sin ^{2} \theta-\left(\rho_{2}-\rho_{4}\right)} .
$$


By using (27), we obtain

$$
\begin{aligned}
\pm\left(\xi_{1}-\xi_{0}\right) & =\int \frac{d \varphi}{\sqrt{\left(\varphi-\rho_{1}\right)\left(\varphi-\rho_{2}\right)\left(\varphi-\rho_{3}\right)\left(\varphi-\rho_{4}\right)}} \\
& =\frac{2}{\sqrt{\left(\rho_{1}-\rho_{3}\right)\left(\rho_{2}-\rho_{4}\right)}} \int \frac{d \theta}{\sqrt{1-m^{2} \sin ^{2} \theta}},
\end{aligned}
$$

$$
\psi_{8}(t, x)=\left[\frac{\rho_{2}\left(\rho_{1}-\rho_{4}\right)\left(-c_{2} / 2 l^{2}\right)^{-1 / 4} \operatorname{sn}^{2}\left(\sqrt{\left(\rho_{1}-\rho_{3}\right)\left(\rho_{2}-\rho_{4}\right)} / 2\left(\left(-c_{2} / 2 l^{2}\right)^{1 / 4} \xi-\xi_{0}\right), m\right)-\rho_{1}\left(\rho_{2}-\rho_{4}\right)}{\left(\rho_{1}-\rho_{4}\right) \operatorname{sn}^{2}\left(\sqrt{\left(\rho_{1}-\rho_{3}\right)\left(\rho_{2}-\rho_{4}\right)} / 2\left(\left(-c_{2} / 2 l^{2}\right)^{1 / 4} \xi-\xi_{0}\right), m\right)-\left(\rho_{2}-\rho_{4}\right)}-\frac{l^{2}}{3}\right] e^{i\left(\alpha t-\int_{a}^{x}\left(\mu^{2} c_{1}+\alpha^{2}\right) \beta(\varsigma) d \varsigma+\theta_{0}\right)},
$$

where $m^{2}=\left(\rho_{1}-\rho_{4}\right)\left(\rho_{2}-\rho_{3}\right) /\left(\rho_{1}-\rho_{3}\right)\left(\rho_{2}-\rho_{4}\right)$. where $\xi=\mu\left(t-2 \alpha \int_{a}^{x} \beta(\varsigma) d \varsigma\right)$.

When $\rho_{3}<\varphi<\rho_{2}$, then we make the transformation

$$
\varphi=\frac{\rho_{4}\left(\rho_{2}-\rho_{3}\right) \sin ^{2} \theta-\rho_{3}\left(\rho_{2}-\rho_{4}\right)}{\left(\rho_{2}-\rho_{3}\right) \sin ^{2} \theta-\left(\rho_{2}-\rho_{4}\right)} .
$$

Similarly, we can obtain the solution of Equation (1):

$$
\psi_{9}(t, x)=\left[\frac{\rho_{4}\left(\rho_{2}-\rho_{3}\right)\left(-c_{2} / 2 l^{2}\right)^{-1 / 4} \operatorname{sn}^{2}\left(\sqrt{\left(\rho_{1}-\rho_{3}\right)\left(\rho_{2}-\rho_{4}\right)} / 2\left(\left(-c_{2} / 2 l^{2}\right)^{1 / 4} \xi-\xi_{0}\right), m\right)-\rho_{3}\left(\rho_{2}-\rho_{4}\right)}{\left(\rho_{2}-\rho_{3}\right) \operatorname{sn}^{2}\left(\sqrt{\left(\rho_{1}-\rho_{3}\right)\left(\rho_{2}-\rho_{4}\right)} / 2\left(\left(-c_{2} / 2 l^{2}\right)^{1 / 4} \xi-\xi_{0}\right), m\right)-\left(\rho_{2}-\rho_{4}\right)}-\frac{l^{2}}{3}\right] e^{i\left(\alpha t-\int_{a}^{x}\left(\mu^{2} c_{1}+\alpha^{2}\right) \beta(\varsigma) d \varsigma+\theta_{0}\right)} .
$$

Case 8. $D_{2} D_{3} \geq 0$ and $D_{4}<0 . G(\varphi)$ has two different real roots and a pair of conjugate complex roots, namely,

$$
G(\varphi)=\left(\varphi-\rho_{1}\right)\left(\varphi-\rho_{2}\right)\left[\left(\varphi-\rho_{3}\right)^{2}+\rho_{4}^{2}\right]
$$

where $\rho_{1}, \rho_{2}, \rho_{3}$, and $\rho_{4}$ are real constants, $\rho_{1}>\rho_{2}$ and $\rho_{4}>0$

We make the following transformation:

$$
\varphi=\frac{\varepsilon_{1} \cos \theta+\varepsilon_{2}}{\varepsilon_{2} \cos \theta+c_{4}}
$$

where $\varepsilon_{1}=1 / 2\left(\rho_{1}+\rho_{2}\right) \varepsilon_{3}-1 / 2\left(\rho_{1}-\rho_{2}\right) \varepsilon_{4}, \varepsilon_{2}=1 / 2\left(\rho_{1}+\rho_{2}\right)$ $c_{4}-1 / 2\left(\rho_{1}-\rho_{2}\right) \varepsilon_{3}, \varepsilon_{3}=\rho_{1}-\rho_{3}-\rho_{4} / m_{1}, \varepsilon_{4}=\rho_{1}-\rho_{3}-\rho_{4} m_{1}$ , $E=\left(\rho_{4}^{2}+\left(\rho_{1}-\rho_{3}\right)\left(\rho_{2}-\rho_{3}\right)\right) / \rho_{4}\left(\rho_{1}-\rho_{2}\right)$, and $m_{1}=E \pm$ $\sqrt{E^{2}+1}$.
By using (27), we obtain

$$
\begin{aligned}
\xi_{1}-\xi_{0} & =\int \frac{d \varphi}{\sqrt{ \pm\left(\varphi-\rho_{1}\right)\left(\varphi-\rho_{2}\right)\left(\left(\varphi-\rho_{3}\right)^{2}+\rho_{4}^{2}\right)}} \\
& =\frac{2 m_{1} m_{2}}{\sqrt{\left(\mp 2 \rho_{4} m_{1}\left(\rho_{1}-\rho_{2}\right)\right)}} \int \frac{d \theta}{\sqrt{1-m_{2}^{2} \sin ^{2} \theta}},
\end{aligned}
$$

where $m_{2}^{2}=2 /\left(1+m_{1}^{2}\right)$.

From Equation (61) and the definition of Jacobian elliptic function, we obtain

$$
\cos \theta=\operatorname{cn}\left(\frac{\sqrt{\mp 2 \rho_{4} m_{1}\left(\rho_{1}-\rho_{2}\right)}}{2 m_{1} m_{2}}\left(\xi_{1}-\xi_{0}\right), m_{2}\right) .
$$

Combining Equation (62) with the expression (60), we can give the solution of Equation (1): 


$$
\psi_{10}(t, x)=\frac{a_{4}^{-1 / 4}\left[\varepsilon_{1} \operatorname{cn}\left(\sqrt{\mp 2 \rho_{4} m_{1}\left(\rho_{1}-\rho_{2}\right)} / 2 m_{1} m_{2}\left(\left(-c_{2} / 2 l^{2}\right)^{1 / 4} \mu\left(t-2 \alpha \int_{a}^{x} \beta(\varsigma) d \varsigma\right)-\xi_{0}\right), m_{2}\right)+\varepsilon_{2}\right]}{\varepsilon_{3} \operatorname{cn}\left(\sqrt{\mp 2 \rho_{4} m_{1}\left(\rho_{1}-\rho_{2}\right)} / 2 m_{1} m_{2}\left(\left(-c_{2} / 2 l^{2}\right)^{1 / 4} \mu\left(t-2 \alpha \int_{a}^{x} \beta(\varsigma) d \varsigma\right)-\xi_{0}\right), m_{2}\right)+\varepsilon_{3}} e^{i\left(\int_{a}^{x}\left(\mu^{2} c_{1}+\alpha^{2}\right) \beta(\varsigma) d \varsigma+\theta_{0}\right)},
$$

which is an elliptic double periodic function solution.

Case 9. $D_{2} D_{3} \leq 0$ and $D_{4}>0 . G(\varphi)$ has two pairs of conjugate complex roots, namely,

$$
G(\varphi)=\left[\left(\varphi-\rho_{1}\right)^{2}+l_{2}^{2}\right]\left[\left(\varphi-\rho_{2}\right)^{2}+l_{2}^{2}\right]
$$

where $\rho_{1}, \rho_{2}, l_{1}$, and $l_{2}$ are real constants and $l_{1} \geq l_{2}>0$.

We make the following transformation:

$$
\varphi=\frac{\varepsilon_{1} \tan \theta+\varepsilon_{2}}{\varepsilon_{3} \tan \theta+\varepsilon_{4}},
$$

where $\varepsilon_{1}=\rho_{1} \varepsilon_{3}+l_{1} \varepsilon_{4}, \varepsilon_{2}=\rho_{1} \varepsilon_{4}-l_{1} \varepsilon_{3}, \varepsilon_{3}=-l_{1}-l_{2} / m_{1}, \varepsilon_{4}=$ $\rho_{1}-\rho_{2}, \quad E=\left(\left(\rho_{1}-\rho_{2}\right)^{2}+l_{1}^{2}+l_{2}^{2}\right) / 2 l_{1} l_{2}, \quad$ and $\quad m_{1}=E+$ $\sqrt{E^{2}-1}$.
By using (27), we obtain

$$
\begin{aligned}
\xi_{1}-\xi_{0} & =\int \frac{d \varphi}{\sqrt{\left[\left(\varphi-\rho_{1}\right)^{2}+l_{2}^{2}\right]\left[\left(\varphi-\rho_{2}\right)^{2}+l_{2}^{2}\right]}} \\
& =\frac{\varepsilon_{3}^{2}+\varepsilon_{4}^{2}}{l_{2} \sqrt{\left(\varepsilon_{3}^{2}+\varepsilon_{4}^{2}\right)\left(m_{1}^{2} \varepsilon_{3}^{2}+\varepsilon_{4}^{2}\right)}} \int \frac{d \theta}{\sqrt{1-m_{2}^{2} \sin ^{2} \theta}},
\end{aligned}
$$

where $m_{2}^{2}=\left(m_{1}^{2}-1\right) / m_{1}^{2}$.

From (65) and the definition of Jacobian elliptic function, we obtain

$$
\begin{aligned}
& \sin \theta=\operatorname{sn}\left(\frac{l_{2} \sqrt{\left(\varepsilon_{3}^{2}+\varepsilon_{4}^{2}\right)\left(m_{1}^{2} \varepsilon_{3}^{2}+\varepsilon_{4}^{2}\right)}}{\varepsilon_{3}^{2}+\varepsilon_{4}^{2}}\left(\xi_{1}-\xi_{0}\right), m_{2}\right), \\
& \cos \theta=\operatorname{cn}\left(\frac{l_{2} \sqrt{\left(\varepsilon_{3}^{2}+\varepsilon_{4}^{2}\right)\left(m_{1}^{2} \varepsilon_{3}^{2}+\varepsilon_{4}^{2}\right)}}{\varepsilon_{3}^{2}+\varepsilon_{4}^{2}}\left(\xi_{1}-\xi_{0}\right), m_{2}\right) .
\end{aligned}
$$

Combining Equations (67) and (68) with expression (65), we can give the solution of Equation (1)

$$
\psi_{11}(t, x)=\left[\frac{\left(-c_{2} / 2 l^{2}\right)^{-1 / 4} \varepsilon_{1} \operatorname{sn}\left(\mu\left(\left(-c_{2} / 2 l^{2}\right)^{1 / 4} \xi-\xi_{0}\right), m_{2}\right)+\left(-c_{2} / 2 l^{2}\right)^{-1 / 4} \varepsilon_{2} \operatorname{cn}\left(\mu\left(\left(-c_{2} / 2 l^{2}\right)^{1 / 4} \xi-\xi_{0}\right), m_{2}\right)}{\varepsilon_{3} \operatorname{sn}\left(\mu\left(\left(-c_{2} / 2 l^{2}\right)^{1 / 4} \xi-\xi_{0}\right), m_{2}\right)+\varepsilon_{4} \operatorname{cn}\left(\mu\left(\left(-c_{2} / 2 l^{2}\right)^{1 / 4} \xi-\xi_{0}\right), m_{2}\right)}-\frac{l^{2}}{3}\right] e^{i\left(\alpha t-\int_{a}^{x}\left(\mu^{2} c_{1}+\alpha^{2}\right) \beta(s) d c+\theta_{0}\right)},
$$

which is an elliptic double periodic function solution, where $\xi=\mu\left(t-2 \alpha \int_{a}^{x} \beta(\varsigma) d \varsigma\right)$.

Remark 4. In this section, we mainly discuss the solution $\psi(t$ $, x)$ of Equation (1). Similarly, we can easily obtain the solution $\phi(t, x)$ of Equation (1) through their relationship $\Psi=\nmid \Phi$.

\section{Conclusion}

In the paper, the planar dynamical system method and the complete discrimination system method are applied to the CNLS equations with variable coefficients for constructing different types of traveling wave solutions. The complex periodic wave solutions, implicit analytical solutions, complex hyperbolic function solutions, and complex Jacobian elliptic function solutions of the CNLS equations with variable coefficients are successfully investigated by two different methods. Moreover, phase portraits are drawn with the help of Maple software. New solutions of the CNLS equations with variable coefficients have not been reported in previous literatures, such as complex Jacobian elliptic function solutions and implicit analytical solutions.

\section{Data Availability}

No data were used to support this study. 


\section{Conflicts of Interest}

The author declares that there is no conflict of interest regarding the publication of this paper. The authors declare no conflict interest.

\section{Acknowledgments}

This work was supported by the Scientific Research Funds of Chengdu University under grant No. 2081920034 and Institutions of Higher Education of Sichuan Province under grant No. MSSB-2021-13.

\section{References}

[1] D. Nath, N. Saha, and B. Roy, "Stability of $(1+1)$-dimensional coupled nonlinear Schrödinger equation with elliptic potentials," The European Physical Journal Plus, vol. 133, no. 12, pp. 504-518, 2018.

[2] Y. Yang, T. Suzuki, and J. Wang, "Backlund transformation and localized nonlinear wave solutions of the nonlocal defocusing coupled nonlinear Schrodinger equation," Communications in Nonlinear Science and Numerical Simulation, vol. 95, article 105626, 2021.

[3] B. Gao and Y. Wang, "Complex wave solutions described by a (3+1)-dimensional coupled nonlinear Schrodinger equation with variable coefficients," Optik, vol. 227, article 166029, 2021.

[4] H. Zhang, X. Meng, T. Xu, L. L. Li, and B. Tian, "Interactions of bright solitons for the $(2+1)$-dimensional coupled nonlinear Schrödinger equations from optical fibres with symbolic computation," Physica Scripta, vol. 75, no. 4, pp. 537-542, 2007.

[5] A.-M. Wazwaz, "Optical bright and dark soliton solutions for coupled nonlinear Schrödinger (CNLS) equations by the variational iteration method," Optik, vol. 207, article 164457, 2020.

[6] H. I. Abdel-Gawad, A. Biswas, A. S. Alshomrani, and M. Belic, "Optical solitons and stability analysis with coupled nonlinear Schrodinger's equations having double external potentials," Results in Physics, vol. 15, article 102707, 2019.

[7] W. Liu, N. Pan, L. Huang, and M. Lei, "Soliton interactions for coupled nonlinear Schrödinger equations with symbolic computation," Nonlinear Dynamics, vol. 78, no. 1, pp. 755-770, 2014.

[8] S. Nandy and A. Barthakur, "Dark-bright soliton interactions in coupled nonautonomous nonlinear Schrodinger equation with complex potentials," Chaos, Solitons \& Fractals, vol. 143, article 110560, 2021.

[9] F. Wu and J. Li, "Dynamics of the smooth positons of the coupled nonlinear Schrodinger equations," Applied Mathematics Letters, vol. 103, article 106218, 2020.

[10] Y. Zhang, C. Yang, W. Yu, M. Mirzazadeh, Q. Zhou, and W. Liu, "Interactions of vector anti-dark solitons for the coupled nonlinear Schrödinger equation in inhomogeneous fibers," Nonlinear Dynamics, vol. 94, no. 2, pp. 1351-1360, 2018.

[11] R. M. EI-Shiekh and M. Gaballah, "Solitary wave solutions for the variable-coefficient coupled nonlinear Schrödinger equations and Davey-Stewartson system using modified sineGordon equation method," Journal of Ocean Engineering and Science, vol. 5, pp. 180-185, 2020.
[12] L. Han, Y. Huang, and H. Liu, "Solitons in coupled nonlinear Schrodinger equations with variable coefficients," Communications in Nonlinear Science and Numerical Simulation, vol. 19, no. 9, pp. 3063-3073, 2014.

[13] X. Liu, L. Zhao, L. Duan, Z. Yang, and W. Yang, "Asymmetric $\mathrm{W}$-shaped and M-Shaped soliton pulse generated from a weak modulation in an exponential dispersion decreasing fiber," Chinese Physics B, vol. 26, no. 12, article 120503, 2017.

[14] X. Wang and X. Hu, "Interactions among periodic optical solitons for the variable coefficient coupled nonlinear Schrodinger equations," Optik, vol. 226, no. 1, article 165621, 2021.

[15] B. He and Q. Meng, "Bifurcations and new exact travelling wave solutions for the Gerdjikov-Ivanov equation," Communications in Nonlinear Science and Numerical Simulation, vol. 15, no. 7, pp. 1783-1790, 2010.

[16] L. Du, Y. Sun, and D. Wu, "Bifurcations and solutions for the generalized nonlinear Schrödinger equation," Physics Letters A, vol. 383, no. 36, article 126028, 2019.

[17] Z. Li, T. Han, and C. Huang, "Bifurcation and new exact traveling wave solutions for time-space fractional Phi-4 equation," AIP Advances, vol. 10, no. 11, article 115113, 2020.

[18] Z. Zhang, Z. Liu, X. Miao, and Y. Chen, "Qualitative analysis and traveling wave solutions for the perturbed nonlinear Schrödinger equation with Kerr law nonlinearity," Physics Letters A, vol. 375, pp. 1275-1280, 2011.

[19] Z. Li and T. Han, "Bifurcation and exact solutions for the (2+1)-dimensional conformable time-fractional Zoomeron equation," Advances in Difference Equations, vol. 2020, 2020.

[20] T. Han, Z. Li, and X. Zhang, "Bifurcation and new exact traveling wave solutions to time-space coupled fractional nonlinear Schrodinger equation," Physics Letters A, vol. 395, article 127217, 2021.

[21] Y. Xie, Z. Yang, and L. Li, "New exact solutions to the high dispersive cubic-quintic nonlinear Schrodinger equation," Physics Letters A, vol. 382, no. 36, pp. 2506-2514, 2018.

[22] Y. Xie, L. Li, and Y. Kang, "New solitons and conditional stability to the high dispersive nonlinear Schrödinger equation with parabolic law nonlinearity," Nonlinear Dynamics, vol. 103, no. 1, pp. 1011-1021, 2021.

[23] L. Li, Y. Xie, and S. Zhu, "New exact solutions for a generalized KdV equation," Nonlinear Dynamics, vol. 92, no. 2, pp. 215$219,2018$. 